\title{
Acquired Factor V Inhibitor in a Cancer Patient: Failure of Immunosuppressive and Rituximab Treatment
} \author{
Tognini $^{2}$ and Elisa Grifoni ${ }^{3}$ \\ ${ }^{1}$ Azienda Ospedaliero, Universitaria Careggi, Italy \\ ${ }^{2}$ Piombino, Azienda Sanitaria Nordovest, Italy \\ ${ }^{3}$ Department of Experimental and Clinical Medicine, University of Florence, Italy
}

Daniela Poli ${ }^{1 *}$, Fabio Pini' ${ }^{2}$, Niccolò Maggini ${ }^{1}$, Anna Paola Cellai ${ }^{1}$, AgatinaAlessandrello Liotta ${ }^{1}$, Annalisa

Submission: March 30, 2017; Published: May 26, 2017

*Corresponding author: Daniela Poli, SOD Malattie Aterotrombotiche, AOU Careggi, Largo Brambilla, 3-50134 Firenze, Italy, Tel: 0039-055-7945453; Fax: 0039-055-7946218; Email: polida@aou-careggi.toscana.it

\begin{abstract}
Acquired factor $\mathrm{V}$ inhibitor is a rare cause of factor $\mathrm{V}$ deficiency, with an overall good prognosis even if the clinical spectrum varies widely. Due to the rarity of the disease, there is scarce information on treatment that is mainly based on immunosuppressive agents. Recently, the use of the anti-CD20 monoclonal antibody rituximab has been associated with a good clinical efficacy in the few reported cases. We describe here the case of a 78 year-old woman with a mesenchimal pleural neoplasm, who developed a mild bleeding diathesis with severe prolongation of prothrombin time and activated partial thromboplastin time due to the presence of acquired factor $\mathrm{V}$ inhibitor. Immunosuppressive treatment with prednisone, cyclophosphamide and rituximab failed to correct coagulopathy. Notwithstanding the severe prolongation of basal coagulation tests and of Factor V deficiency, the patient remained asymptomatic for bleeding and died 2 years later for the progression of the pleural neoplasm.
\end{abstract}

Keywords: Factor V; Acquired inhibitor; Rituximab

Abbreviations: PT: Prothrombin Time; aPTT: activated Partial Thromboplastin Time; FV: Factor V

\section{Introduction}

Acquired factor $\mathrm{V}$ (FV) inhibitor is a rare cause of $\mathrm{FV}$ deficiency, with less than 200 cases reported in the literature, and with an estimated incidence is 0.09-0.29 per million person-years [1]. Factor $\mathrm{V}$ inhibitor has been associated with a number of conditions, including antibiotic use, sepsis, autoimmune diseases and malignancies. In particular among patients with FV inhibitors, cancer was detected in $9-16 \%$ of cases [2]. In addition, factor $\mathrm{V}$ inhibitors have been described after exposure to bovine thrombin, used as topical hemostatic agent in surgery [1].

The overall prognosis is good, even if the clinical spectrum varies widely from the more common occurrence of mild bleedings or absence of symptoms to the less frequent severe bleeding events. The diagnosis of FV inhibitor is based on increased prothrombin time (PT), activated partial thromboplastin time (aPTT), decreased FV levels, and confirmed by the presence of FV inhibitors titrated using the Bethesda method. The prognosis is closely related to the underlying disease and the eradication of the triggering factor should be a first goal of the treatment. There is scarce information on treatment, and recommendations are extrapolated form the more common management of factor VIII inhibitors, which is mainly based on immunosuppressive agents. In the last few years the use of the anti-CD20 monoclonal antibodyrituximab has been reported in 8 cases [3-9], demonstrating a good clinical efficacy in eradicating the coagulopathy in all the reported cases. However, due to the rarity of the disease, no clinical trials are available to establish the efficacy and safety of this treatment, and clinicians have to rely only on case reports [6]. We describe here the case of a patient with acquired FV inhibitor, who failed to respond to rituximab treatment.

\section{Case Report with Results}

A 78 years old woman with neither familiar nor personal history of hemorrhagic disorders underwent surgery for the removal of a mesenchimal malignant neoplasm of the thoracic 
wall. Preoperative basal plasma coagulation tests, PT and aPTT, were normal. The patient was discharged 7 days after surgery without complications. During surgery no bovine thrombin preparations were used. Three weeks after discharge a voluminous hematoma of the surgical wound $(12 \times 8 \times 6 \mathrm{~cm})$ spontaneously developed. Needle aspiration of the hematoma was performed and a compressive medication was applied. Few days later the hematoma recurred, but at aspiration minimal sero-hematic fluid was drawn and it was not possible to reduce the sub-cutaneous mass due to hematoma organization. Blood samples results showed: hemoglobin $8.2 \mathrm{gr} / \mathrm{dL}$, platelets 291.000, PT14\% and aPTT>240", fibrinogen $402 \mathrm{mg} / \mathrm{dL}$. The patient did not show other bleeding manifestations. For this reason she came to our attention for evaluation. PT and aPTT tests alterations confirmed that both intrinsic and extrinsic coagulation pathways were involved, suggesting the presence of an acquired inhibitor of a common pathway coagulation factor. Coagulation factor activities were not measurable on undiluted patient's sample, therefore laboratory tests were repeated by using dilution (1/10), which demonstrated a return to near normal levels of all coagulation factors with the exception of factor $\mathrm{V}$, which remained undetectable. Factor $\mathrm{V}$ inhibitor was suspected and confirmed with a titre of 1388 Bethesda Units/mL.

At diagnosis the patient was asymptomatic for bleeding, hemoglobin levels were stable, and treatment with prednisone $1 \mathrm{mg} / \mathrm{Kg} /$ day of body weight was started. After 2 weeks of treatment laboratory data were unchanged, the patient was clinically stable with the exception of rare cutaneous bruises. Treatment with cyclophosphamide $50 \mathrm{mg} /$ day was added, but 2 weeks later laboratory findings were still unchanged. Treatment with prednisone $1 \mathrm{mg} / \mathrm{kg} /$ day was maintained and cyclophosphamide dosage was increased up to $200 \mathrm{mg}$ /day. Few days later the patient complained a back pain with diffusion to the right leg. A tomography showed a large hematoma of the obturator muscle. PT and aPTT levels persisted unmodified, factor $\mathrm{V}$ levels were still undetectable, and factor V inhibitor title was reduced to 195 Bethesda Units/ $\mathrm{mL}$. Cyclophosphamide treatment was stopped, and a course of treatment with rituximab was started $(375 \mathrm{mg} / \mathrm{m} 2 /$ week for 4 weeks). Also this treatment was not able to eradicate the inhibitor, and results of coagulation tests after one month of rituximab treatment demonstrated: unmodified PT and aPTT levels, undetectable factor $\mathrm{V}$ levels, and factor $\mathrm{V}$ inhibitor title further reduced to $112 \mathrm{U}$ Bethesda/ml. A slower reduction in factor $\mathrm{V}$ inhibitor title was measured during follow-up, reaching a title of 46 Bethesda Units/mL, with factor $V$ levels of $3.5 \% 2$ months after the conclusion of rituximab treatment. Eight months after the diagnosis the patient developed severe osteoporosis with a spontaneous vertebral fracture; therefore, prednisone was progressively tapered down to $5 \mathrm{mg}$ /day. Slight progressive reduction in basal coagulation tests was recorded, according with a reduction of factor $\mathrm{V}$ inhibitor to 5 Bethesda
Units/mL measured one month before death (Figure 1). The patient died 2 years after the diagnosis, as a consequence of the local progression of the neoplasm, without recovery from coagulopathy and without clinically relevant bleeding events.

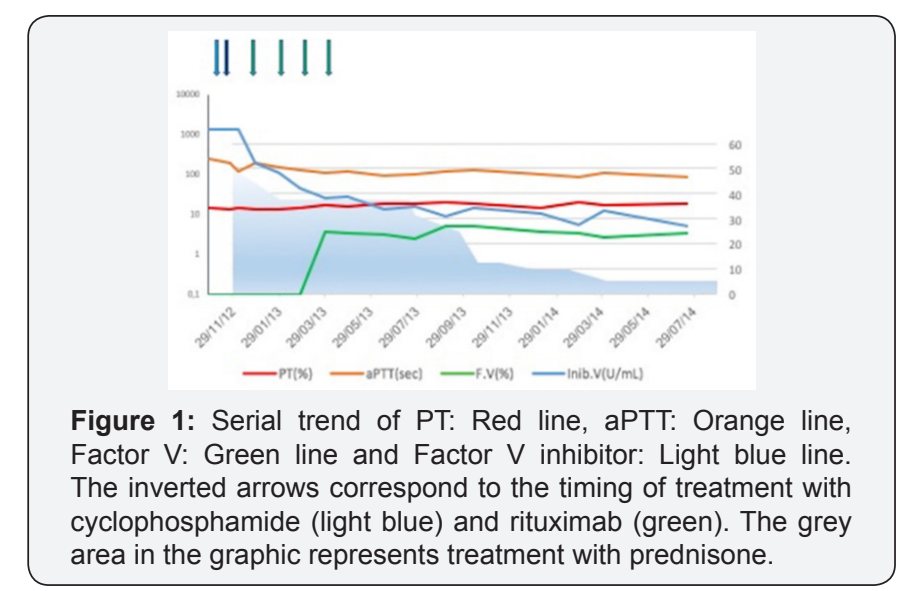

\section{Discussion}

At best of our knowledge, 8 patients with acquired FV inhibitors treated with rituximab have been described in the literature, and all showed good response to treatment. In the described patients a 4-week course of standard doses of rituximab was conducted, with the exception of one case who received 8 weeks of treatment to achieve clinical response [8]. Unfortunately, in our case a 4 weeks of rituximab at standard doses, as well as steroidal and cyclophosphamide immunosuppressive treatment, did not correct the coagulopathy. However, as reported, our patient was asymptomatic for bleedings, and the use of immunosuppressive treatment is not established or recommended as standard therapy in non bleeding patients. Therefore, further immunosuppressive treatment was avoided with a progressive tapering of prednisone with the aim of minimizing adverse effects. Factor V inhibitor levels maintained stable, despite the stopping of immunosuppressive treatment and steroids tapering.

In contrast with other coagulation factors inhibitors, particularly those of factor VIII, the level of factor V inhibitor does not correlate with the severity of clinical bleeding [1]. In our case, as frequently reported in these patients, the clinical features of the disease were mild, notwithstanding the severe prolongation of both basal plasma coagulation tests PT and aPTT. As a matter of fact, it is quite common that the severity of the laboratory findings rises clinical alarm in contrast to the relatively asymptomatic patients, confirming that the risk of bleeding does not correlate with the entity of PT and aPTT prolongation, nor with factor $\mathrm{V}$ and factor $\mathrm{V}$ inhibitor levels, with a relative discrepancy between laboratory and clinical manifestations. In this clinical contest the use of aggressive immunosuppressive therapy should be carefully evaluated, bearing in mind the large spectrum of associated adverse events. In our patient, after 8 months of steroidal treatment, 
a severe osteoporosis was developed, and the patient experienced a spontaneous vertebral fracture. Therefore, case reports suggest that rituximab treatment could be useful in patients with factor $\mathrm{V}$ inhibitors; however, due to the usually mild clinical manifestations, the use of aggressive immunosuppressive regimens should be carefully evaluated.

\section{References}

1. Franchini M, Lippi G (2011) Acquired factor V inhibitors: a systematic review. J Thromb Thrombolysis 31(4): 449-457.

2. Ahmadinejad M, Roushan N (2013) Acquired factor V inhibitor developing in a patient with esophageal squamous cell carcinoma. Blood Coagul Fibrinolysis 24(1): 97-99.

3. Lian EC, Tzakis AG, Andrews D (2004) Response of factor V inhibitor to rituximab in a patient who received liver transplantation for primary biliary cirrhosis. Am J Hematol 77(4): 363-365.

4. Perdekamp MT, Rubenstein DA, Jesty J, Hultin MB (2006) Platelet factor $\mathrm{V}$ supports hemostasis in a patient with an acquired factor $\mathrm{V}$ inhibitor, as shown by prothrombinase and tenase assays. Blood Coagul Fibrinolysis 17(7): 593-597.

5. Lebrun A, Leroy-Matheron C, Arlet J-B, Bartolucci P, Michel M (2008) Successful treatment with rituximab in a patient with an acquired factor V inhibitor. Am J Hematol 83(2): 163-164.

6. Navarrete MA, van der Meer FJ, Damiani G, Diaz A, Eikenboom J (2012) The use of rituximab therapy in patients with acquired factor $V$ inhibitors. Am J Hematol 87(8): 826-827.

7. John ES, Patel MD, Hajdenberg J (2015) Refractory epistaxis due to severe factor V deficiency with inhibitor. Case Rep Hematol 2015: 603402.

8. Ma ES, Liang RH, Chu KM, Lau GK (2015) Complete response of acquired FV inhibitor to rituximab. Int J Hematol 101(4): 421-422.

9. Patel MD, Hajdenberg J (2016) Successful treatment of chronic recurrent life-threating bleeding due to an acquired factor $\mathrm{V}$ inhibitor with rituximab and steroids. Haemophilia 22(3): e231-e232.

\begin{tabular}{|l|}
\hline \multicolumn{1}{|c|}{ Your next submission with Juniper Publishers } \\
will reach you the below assets \\
- Quality Editorial service \\
- Swift Peer Review \\
- Reprints availability \\
- E-prints Service \\
- Manuscript Podcast for convenient understanding \\
- Global attainment for your research \\
- Manuscript accessibility in different formats \\
( Pdf, E-pub, Full Text, Audio) \\
- Unceasing customer service \\
Track the below URL for one-step submission \\
https://juniperpublishers.com/online-submission.php \\
\hline
\end{tabular}

